\title{
Specific IgE against the house dust mite Suidasia pontifica as a risk factor for asthma and allergies in the tropics
}

\author{
Jennifer Maries G. Yap ${ }^{1}$, Maricar W. Ching ${ }^{1,4}$, Rogelio S. Cruz ${ }^{1,4}$, E John Donnie A. Ramos ${ }^{1,2,3 *}$ \\ ${ }^{1}$ The Graduate School; ${ }^{2}$ Department of Biological Sciences, College of Science; \\ ${ }^{3}$ Research Center for the Natural and Applied Sciences, University of Santo Tomas \\ ${ }^{4}$ Centro Escolar University \\ Manila, PHILIPPINES
}

Background. Suidasia pontifica (Sp) mites are medically significant because of their ability to produce potent allergens causing serious diseases like allergic asthma, dermatitis and rhinitis. Its clinical relevance in the Filipino population has never been characterized.

Methods. The immunoglobulin E-binding activity of allergens from the house dust mite (HDM) Sp was determined in 258 allergic cases and 379 non-atopic Filipino subjects using enzyme-linked immunosorbent assay (ELISA). Correlation between $S p$-specific IgE and total IgE concentration was determined. Likewise, multiple regression analysis was used to determine the effect of Sp-specific IgE to the total IgE concentration of allergic patients.

Results. Sensitization to Sp allergens was demonstrated in 39\% (100/258) of Filipino allergic cases tested. A significant correlation was observed between total IgE and Sp-specific IgE level among allergic patients ( $p=0.038$ ). In addition, an increase in $S p$-specific IgE is expected to increase the total IgE concentration as well $(p=0.000)$. Sp-specific IgE is demonstrated as an independent risk factor for the development of allergic diseases ( $\mathrm{OR}=3.12$, $\mathrm{CI}=2.16-5.41)$.

Conclusion. The results obtained in this study suggest that the HDM species $S p$ is an important source of allergens that trigger sensitization in various allergic cases. Thus, the incorporation of $S p$ allergens in the panel of diagnostic allergens for HDM allergy and allergic rhinitis is highly recommended.

Keywords: allergy, asthma, total IgE, HDM-specific IgE

*To whom correspondence should be addressed jdaramos@mnl.ust.edu.ph / ramosjda@yahoo.com

\section{INTRODUCTION}

Allergic diseases such as asthma, rhinitis, and dermatitis have become a major worldwide epidemic problem with increasing prevalence for 
the last $20-30$ years and affecting $10-40 \%$ of the world population [1-4]. Allergy is a hypersensitivity reaction of the immune system to commonly inhaled proteins called allergens characterized by the production of elevated levels of allergen-specific immunoglobulin E (IgE) [5-6]. Although allergen sources are very diverse, $60-90 \%$ of allergies are triggered by allergens from house dust mites (HDMs) [7]. Exposure to HDM allergens is a risk factor in the development of asthma, and it may exacerbate allergic symptoms [8]. More than 90\% of children with asthma develop specific IgE sensitization against allergens from HDM [911]. The HDM species Dermatophagoides farinae (Df), Dermtophagoides pteronyssinus $(D p)$, and Blomia tropicalis (Bt) are the most clinically important species in tropical and subtropical regions [12-13]. In the Philippines, approximately $80-90 \%$ of the Filipinos are sensitized to these HDM species [14]. Although HDM species $B t, D f$, and $D p$ are important sources of allergens among Filipino allergic patients, an acarofauna study suggests that other HDM species are found in dust samples in houses of allergic patients in Metro Manila [15].

Suidasia pontifica $(S p)$ mites are considered to have medical significance because of their ability to produce potent allergens causing serious diseases like allergic asthma, dermatitis and rhinitis. Suidasia pontifica mites occur mainly in hot climates and are representatives of the acarofauna of tropical countries [16]. An important degree of cross-reactivity has also been established between $S p$ and other common HDM species. Although the allergenicity of $S p$ has been well studied in other populations, its clinical relevance in the Filipino population has never been characterized.

\section{EXPERIMENTAL}

Study design and subjects. The study made use of case-control study design, employing 258 allergic cases and 380 nonallergic controls.
Subjects were recruited from the University of Santo Tomas Hospital (USTH) and clinics from the provinces, as approved by the University of Santo Thomas Hospital Institutional Review Board. Informed consents and assents were obtained from the patients as indicated in the protocol.

Clinical protocol and phenotyping. Five (5) milliliters of blood was extracted by venipuncture from qualified subjects. Blood serum samples were isolated by centrifugation at 10,000 rpm for $10 \mathrm{~min}$. Total serum IgE and HDM-specific IgE concentration were determined using enzyme-linked immunosorbent assay (ELISA). IgE concentration was expressed as IU/mL and was log transformed to obtain normality.

\section{Total IgE enzyme-linked immunosorbent} assay. Enzyme-linked immunosorbent assay was used to determine the total IgE concentrations of the allergic cases and non-allergic controls. Briefly, $10 \mu \mathrm{g} / \mathrm{mL}$ of unlabelled anti-human IgE (Pharmingen, CA, USA) were coated onto ELISA plates (Greiner Bio-One, Germany) overnight at $4^{\circ} \mathrm{C}$ using $50 \mu \mathrm{L}$ of $0.1 \mathrm{M} \mathrm{NaHCO}$, $\mathrm{pH}$ 8.3. Plates were blocked with $1 \%$ BSA (Sigma-Aldrich, Saint Louis, MO, USA) in Phosphate Buffered Saline with $0.05 \%$ Tween 20 (PBS-T) for $1 \mathrm{~h}$ at room temperature. ELISA plates were incubated overnight with $5 \times$ diluted human sera, then for $1 \mathrm{~h}$ at room temperature with biotinylated antihuman IgE (Pharmingen, CA, USA) diluted $1000 \times$ in blocking buffer. Plates were incubated with $2000 \times$ dilution of ExtrAvidin-Alkaline phosphatase conjugate (Sigma-Aldrich, Saint Louis, MO, USA) for $1 \mathrm{~h}$. Finally, colorimetric reaction was performed using $p$-nitrophenyl phosphate (Sigma-Aldrich, Saint Louis, MO, USA). Absorbance was read at $405 \mathrm{~nm}$ on ELISA plate reader (BioTek Instruments Inc, USA).

House dust mite-specific IgE enzyme-linked immunosorbent assay. Enzyme-linked immunosorbent assay (ELISA) was used to evaluate the profile of sensitization of Filipino 
allergic subjects to aqueous Sp extract. Fifty (50) microliter of the aqueous extracts diluted to a final concentration of $10 \mathrm{mg} / \mathrm{mL}$ using $0.1 \mathrm{M}$ NaHCO3 (Sigma-Aldrich, Saint Louis, MO, USA) was coated onto ELISA plates (Corning Costar Inc., NY, USA) overnight at $4^{\circ} \mathrm{C}$. Plates were blocked with 1\% BSA (Sigma-Aldrich, Saint Louis, MO, USA) in Phosphate buffered saline $-0.05 \%$ Tween 20 (PBST) for $1 \mathrm{~h}$ at room temperature. ELISA plates were incubated overnight at $4^{\circ} \mathrm{C}$ with human sera diluted five times in blocking buffer followed by 1-h incubation with biotinylated antihuman IgE (Pharmingen, CA, USA) diluted $1000 \times$ in blocking buffer. Plates were then incubated with 2000x dilution of ExtrAvidin-Alkaline Phosphatase conjugate (Sigma-Aldrich, Saint Louis, MO, USA) for $1 \mathrm{~h}$. Finally, colorimetric reaction was performed using $p$-nitrophenyl phosphate (Sigma-Aldrich, Saint Louis, MO, USA). Plates were washed with $200 \mu \mathrm{L}$ of PBST per well for every addition of reagent.
Absorbance at $405 \mathrm{~nm}$ was determined using the Bio-Tek ELX800 ELISA reader (Tecan, Austria).

Statistical analysis. Data were analyzed using statistical packages, SPSS v.16, Graphpad Prism 6, and MS Excel. Statistical analyses consisted of determinations of mean specific IgE levels, mean and standard deviations of mite allergen concentrations, group comparisons made by using contingency tables, Chi Square analysis, unpaired two-tailed Student's $t$-test, and Pearson correlation test. Categorical data regression analysis and odds ratios (OR) of allergic diseases according to HDM allergen level and allergen sensitization were estimated by the MantelHaenszel statistic. Statistical significance was set at $p$-value $<0.05$.

\section{Results}

A total of 637 allergic and non-atopic subjects whose were included in the study (Table 1). The age distribution in both groups ranged between

Table 1. Demographic Profile of Allergic Cases and Non-atopic Controls

\begin{tabular}{|c|c|c|c|}
\hline & & $\begin{array}{c}\text { Allergic Cases } \\
\quad(n=258)\end{array}$ & $\begin{array}{c}\text { Non-atopic } \\
\text { Controls } \\
(n=379)\end{array}$ \\
\hline \multirow{2}{*}{ Age } & $1-18$ years old & 225 (87\%) & $319(84 \%)$ \\
\hline & 19 and above & $33(12 \%)$ & $60(16 \%)$ \\
\hline \multirow{2}{*}{ Gender } & Male & $119(46 \%)$ & $152(40 \%)$ \\
\hline & Female & 139 (54\%) & $227(60 \%)$ \\
\hline \multirow{2}{*}{ Location } & Urban & 245 (95\%) & 372 (98\%) \\
\hline & Rural & $13(5 \%)$ & $7(2 \%)$ \\
\hline \multirow{7}{*}{ Type of Allergy } & $\mathrm{AA}^{1}$ & $85(33 \%)$ & \multirow{7}{*}{$N A^{4}$} \\
\hline & $A R^{2}$ & $64(25 \%)$ & \\
\hline & $A D^{3}$ & $13(5 \%)$ & \\
\hline & $\mathrm{AA}$ and $\mathrm{AR}$ & $44(17 \%)$ & \\
\hline & $A A$ and $A D$ & 25 (10\%) & \\
\hline & $A R$ and $A D$ & $14(5 \%)$ & \\
\hline & $A A, A R$ and $A D$ & $13(5 \%)$ & \\
\hline \multirow{2}{*}{ Pets } & Yes & $55(21 \%)$ & $114(30 \%)$ \\
\hline & No & 203 (79\%) & $265(70 \%)$ \\
\hline \multirow{2}{*}{ Sleeping in AC Rooms ${ }^{5}$} & Yes & $52(20 \%)$ & 127 (34\%) \\
\hline & No & 206 (80\%) & $252(66 \%)$ \\
\hline
\end{tabular}

${ }^{1} \mathrm{AA}$, allergic asthma; ${ }^{2} \mathrm{AR}$, allergic rhinitis; ${ }^{3} \mathrm{AD}$, atopic dermatitis; ${ }^{4} \mathrm{NA}$, not applicable;

${ }^{5}$ sleeping in air-conditioned rooms 

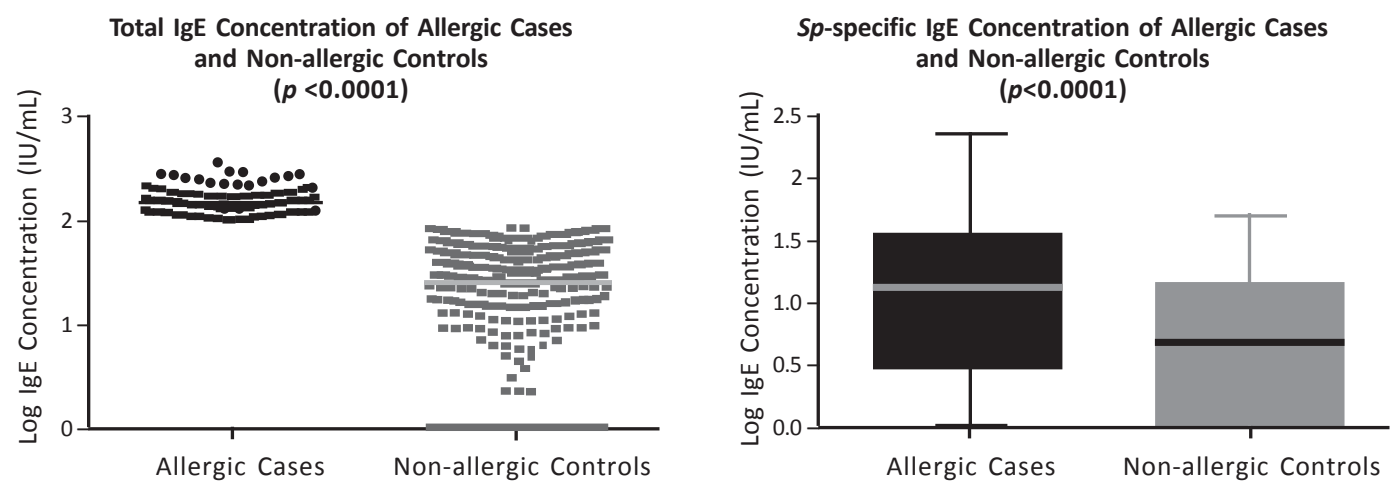

Figure1. Total IgE and Sp-specific IgE levels of 258 allergic cases and 379 non-atopic controls
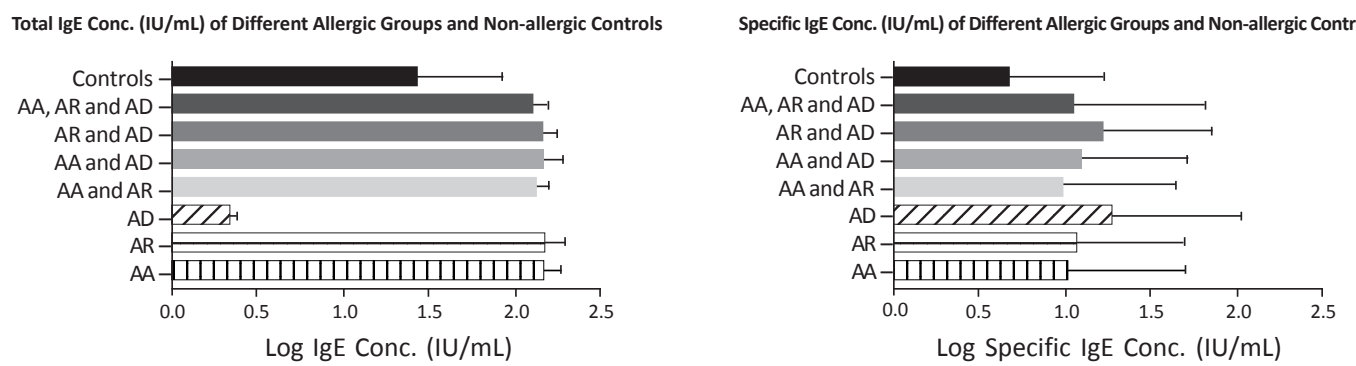

$$
\begin{gathered}
\text { AA, allergic asthma; AR, allergic rhinitis; } A D \text {, atopic dermatitis } \\
\text { AA with AR cases, allergic asthma with allergic rhinitis } \\
\text { AA with AD cases, allergic asthma with atopic dermatitis } \\
\text { AA with } A R, A D \text { cases, allergic asthma with allergic rhinitis and atopic dermatitis }
\end{gathered}
$$

Figure 2. Total IgE and $S p$-specific IgE levels among different allergic groups and non-allergic controls

3 and 46 years old. The mean age in non-atopic controls (12.6 \pm 5.8 years) is slightly higher than in allergic cases (11.8 \pm 5.0 years). Majority of the cases have asthma concurrent with other diseases like rhinitis and atopic dermatitis.

Thirty-nine (39) percent (100/258) of the allergic cases exhibited positive reaction with allergens from $S p$ aqueous extracts using a cut-off value of 20.68, corresponding to mean+1SD of the IgE levels of non-atopic controls. Cases with atopic dermatitis only demonstrated the highest positive reaction, 54\% (7/13), followed by cases with allergic asthma only, 40\% (32/81), and allergic rhinitis only, 38\% (23/61). Unpaired $t$ test showed a significant difference in the total and $S p$ allergen-specific IgE concentration of allergic cases and non-atopic controls tested (Fig. 1). However, there is no significant difference between the total and $S p$ allergenspecific IgE concentration among different allergic groups (Fig. 2).

Correlation between total IgE and Sp-specific IgE concentration was significant regardless of their allergic disease. Furthermore, a significant correlation between the total IgE and Sp-specific igE concentration was observed among cases with allergic rhinitis only. Production of $S p$ specific IgE is an independent risk factor for the development of allergic diseases ( $\mathrm{OR}=3.12$, CI = 2.16-5.41) (Table 2). 
Table 2. Correlation Between Total IgE level and Sp-specific IgE Levels and Regression Analysis of $\mathbf{3 7 9}$ Non-Atopic Control Subjects versus 258 Allergic Cases

\begin{tabular}{l|c|c|c|c}
\hline & & Correlation $^{\mathrm{a}}$ & $p$-value & Odds Ratio $^{\mathrm{b}}(95 \% \mathrm{Cl})$ \\
\hline \multirow{4}{*}{$\begin{array}{l}\text { Total IgE vs. } \\
\text { HDM- specific }\end{array}$} & All Cases & 0.207 & $0.001^{\mathrm{S}}$ & $3.12(2.16-5.41)^{\mathrm{S}}$ \\
\cline { 2 - 5 } IgE & AA only & 0.165 & $0.142^{\mathrm{NS}}$ & $3.21(1.91-5.41)^{\mathrm{S}}$ \\
\cline { 2 - 5 } & $\mathrm{AR}$ only & 0.312 & $0.014^{\mathrm{S}}$ & $3.19(1.79-5.70)^{\mathrm{S}}$ \\
\cline { 2 - 5 } & $\mathrm{AD}$ only & -0.143 & $0.641^{\mathrm{NS}}$ & $5.74(1.87-17.65)^{\mathrm{S}}$ \\
\cline { 2 - 5 } & $\mathrm{AA}$ and AR & 0.215 & $0.162^{\mathrm{NS}}$ & $1.85(.902-3.78)^{\mathrm{NS}}$ \\
\cline { 2 - 5 } & $\mathrm{AA}$ and AD & 0.327 & $0.111^{\mathrm{NS}}$ & $6.22(2.71-14.38)^{\mathrm{S}}$ \\
\cline { 2 - 5 } & $\mathrm{AR}$ and AD & 0.311 & $0.278^{\mathrm{NS}}$ & $2.73(.887-8.43)^{\mathrm{NS}}$ \\
\cline { 2 - 5 } & $\mathrm{AA}, \mathrm{AR}$, and AD & 0.151 & $0.623^{\mathrm{NS}}$ & $3.08(.975-9.71)^{\mathrm{NS}}$ \\
\hline
\end{tabular}

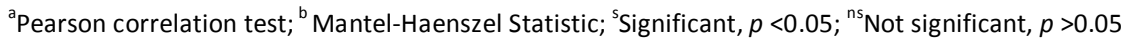

Table 3. Regression Analysis of the Total IgE Concentration and Sp-specific IgE Concentration of Allergic Cases and Non-allergic Controls

\begin{tabular}{|c|c|c|c|c|}
\hline $\begin{array}{l}\text { Dependent Variable: } \\
\text { TOTAL IgE CONC. }\end{array}$ & $\begin{array}{c}\text { Parameter } \\
\text { Estimate }\end{array}$ & $\begin{array}{l}\text { Standard } \\
\text { Error }\end{array}$ & Test Stat & $p$-value \\
\hline \multicolumn{5}{|c|}{ All allergic cases, regardless of atopy status } \\
\hline Intercept & .334 & .022 & 15.243 & .000 \\
\hline$S p$-specific IgE & .276 & .043 & 6.384 & .000 \\
\hline \multicolumn{5}{|l|}{ AA cases only ${ }^{1}$} \\
\hline Intercept & .135 & .020 & 6.885 & .000 \\
\hline Sp-specific IgE & .199 & .043 & 4.643 & .000 \\
\hline \multicolumn{5}{|l|}{ AR cases only ${ }^{2}$} \\
\hline Intercept & .105 & .018 & 5.804 & .000 \\
\hline Sp-specific IgE & .168 & .040 & 4.139 & .000 \\
\hline \multicolumn{5}{|l|}{ AD cases only ${ }^{3}$} \\
\hline Intercept & .019 & .010 & 1.894 & .059 \\
\hline Sp-specific IgE & .080 & .023 & 3.445 & .001 \\
\hline \multicolumn{5}{|l|}{ AA with AR cases ${ }^{4}$} \\
\hline Intercept & .092 & .016 & 5.633 & .000 \\
\hline$S p$-specific IgE & .066 & .039 & 1.700 & .090 \\
\hline \multicolumn{5}{|l|}{ AA with AD cases ${ }^{5}$} \\
\hline Intercept & .034 & .013 & 2.598 & .010 \\
\hline Sp-specific IgE & .146 & .030 & 4.919 & .000 \\
\hline \multicolumn{5}{|l|}{ AA with $A R, A D$ cases $^{6}$} \\
\hline Intercept & .025 & .010 & 2.492 & .013 \\
\hline Sp-specific IgE & .048 & .024 & 2.014 & .045 \\
\hline \multicolumn{5}{|l|}{ 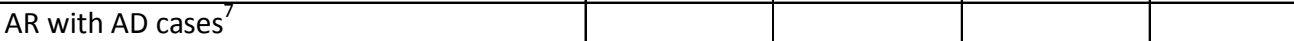 } \\
\hline Intercept & .028 & .010 & 2.702 & .007 \\
\hline$S p$-specific IgE & .045 & .025 & 1.821 & .069 \\
\hline
\end{tabular}

${ }^{1} A A$, allergic asthma; ${ }^{2} A R$, allergic rhinitis; ${ }^{3} A D$, atopic dermatitis; ${ }^{4} A A$ with $A R$ cases, allergic asthma with allergic rhinitis; ${ }^{5} A A$ with $A D$ cases, allergic asthma with atopic dermatitis; ${ }^{6} A A$ with $A R, A D$ cases, allergic asthma with allergic rhinitis and atopic dermatitis; ${ }^{7} A R$ with $A D$ cases, allergic rhinitis with atopic dermatitis 
Table 4. Logistic Regression Analysis of Independent Variables on the Total IgE Concentration of Allergic Cases and Non-allergic Controls

\begin{tabular}{|c|c|c|c|}
\hline $\begin{array}{l}\text { Dependent Variable: Total } \\
\text { IgE Concentration }\end{array}$ & Coefficient & $\begin{array}{c}\text { Standard } \\
\text { Error }\end{array}$ & $p$-value \\
\hline $\mathrm{Age}^{1}$ & -0.322 & 0.239 & 0.178 \\
\hline Gender $^{2}$ & 0.223 & 0.166 & 0.180 \\
\hline Pets $^{3}$ & -0.418 & 0.192 & 0.030 \\
\hline Air-conditioned rooms ${ }^{4}$ & -0.636 & 0.192 & 0.001 \\
\hline
\end{tabular}

An increase in Sp-specific IgE is expected to increase the total IgE concentration as shown in a regression analysis (Table 3). Moreover, the $p$ values of the cases, except those of AA with $\mathrm{AR}$ and $\mathrm{AR}$ with $\mathrm{AD}$ are all less than $5 \%$, which means that $S p$-specific IgE has significant contribution in explaining the variability in the total IgE concentration. The coefficient of estimate of $S p$-specific IgE is the largest for all allergic cases, regardless of their atopy status and those AA cases only second. The coefficient of estimate for all allergic cases is 0.276 , which means that for a 1 -unit increase in $\mathrm{Sp}$-specific IgE concentration, it is expected that there is an approximately 0.276 increase in total IgE concentration, regardless of the atopy status. Further analysis also showed that for a 1-unit increase in Sp-specific IgE concentration, it is expected that there is an approximate 0.199 increase in the total IgE concentration of cases with allergic asthma (Table 3).

Regression analysis using total IgE concentration as dependent variable compared with independent variables such as age, gender, presence of pets and living in AC rooms showed that at least one of the independent variables contribute to the total IgE concentration $(p=0.0002)$. Subjects without pets at home are $65 \%$ less likely to have increased total IgE concentration than those who have pets. Likewise, subjects who are in air conditioned environment most of the time are 53\% less likely to have increased total IgE concentration than those who are not in air-conditioned environment (Table 4).

\section{Discussion}

Allergic diseases such as allergic asthma, allergic rhinitis, and atopic dermatitis caused by sensitization to allergens from mites are a problem throughout the world [11, 17, 18]. House dust mites have been well recognized to play an important role in the pathogenesis of allergic diseases [19-21]. Several studies confirmed that HDM sensitization is a major risk factor for these allergic diseases especially in asthma [22, 23]. Common HDM implicated in allergic diseases, such as $B t, D f$, and $D p$ ), are considered ubiquitous in every household especially in the tropics such as the Philippines. Another predominant species found in tropical and subtropical regions is $S p$, belonging to the superfamily Acaroidea. They are found common in nests of vertebrates and insects and in stored food products [24]. Various studies indicating the allergenic role of Suidasia spp. has been suggested. In Venezuela, it has been implicated in cases of anaphylaxis after ingestion of wheat contaminated mites [25]. In Cartagena, a high percentage of the asthmatic patients evaluated were sensitized to this mite species, indicating the clinical relevance of Suidasia spp. in allergic asthma. Moreover, an important degree of crossreactivity was established between $S p, B t$, and $D$, which are other common sources of allergens implicated in allergic diseases [24]. In one study, more than $70 \%$ of suspected patients with 
allergic rhinitis demonstrated positive reaction when tested to $S p$ extracts, indicating the significant role of $S p$ allergens in the pathogenesis of allergic rhinitis [26]. Locally, an acarofauna survey revealed that $B t, D p, D f$, and $S p$ are the most predominant mite species in the dust samples collected from houses of allergic patients in Metro Manila [14].

The results of this study are in agreement with other epidemiologic observations, which suggest that $70-80 \%$ of patients with asthma are sensitive to HDM [18]. A study by Ramos et al., showed that Filipino allergic patients were also sensitized to other HDM allergen species, namely, $B t, D p$, and $D f$. The results of this study demonstrated that dust mite sensitization is a dominant risk factor for development of allergic diseases among a population living in humid temperate climate which is highly favorable to the development of different HDM species [27]. In this study no significant difference in the total and $S p$-specific IgE concentration among varying ages and sex was observed. However, it is noteworthy that the likelihood of a probable increase in total IgE concentration was observed among those subjects exposed to animals/pets and those in air-conditioned environment than those who were not in the same living conditions. It has been proposed that early exposure to microbial life, irrespective of pet exposure, may favor the development toward Th1 immunity. It could also be that exposure to the environment early in life may have induced tolerance [11, 22].

In a previous study by Puerta et al., the whole body mite extract of $S p$ has at least 14 allergens, suggesting an important allergenic load and sensitizing potential, comparable with that of the more common mite species involved in allergic respiratory diseases [24]. The allergen with an apparent molecular weight of 30-31 kDa was detected by more than $50 \%$ of allergic patients (54.8\%) and can be considered as a major allergen. As have been pointed out, Bt,
$D p$, and $D f$ are main sensitizing HDM among allergic Filipinos [27]. However, this study has contributed to the demonstration of the clinical role of allergens from $S p$ in the development of asthma and other allergic diseases in Filipino population, where $S p$ in the household is considered endemic.

\section{AcKnowledgments}

This study was supported by research grants from the Department of Science and Technology-Accelerated Science and Technology Human Resource Development Program and the Commission on Higher Education-Zonal Research Center. The researchers would like to acknowledge the Research Center for the Natural and Applied Sciences (RCNAS) for housing the experiment, and the University of Santo Tomas Hospital Ethics Review Board for reviewing our protocol. We would also like to thank Mr. Kevin Carl Santos of University of the Philippines for his assistance in the statistical analysis of data.

\section{REFERENCES}

[1] Boyce JA, Broide D, Matsumoto K, Bochner BS. Advances in mechanisms of asthma, allergy, and immunology in 2008. J. Allergy Clin. Immunol. 2009; 123(3):569-574.

[2] Bush RK. Indoor allergens, environmental avoidance, and allergic respiratory disease. Allergy Asthma Proc. 2008; 29(6):575-579.

[3] Holt PG, Thomas WR. Sensitization to airborne environmental allergens: unresolved issues. Nat. Immunol. 2005 Oct.; 6(10):957-960.

[4] Shek LP-c, Chong AR, Soh SE, Cheong N, Teo AS, Yi FC, et al. Specific profiles of house dust mite sensitization in children with asthma and in children with eczema. Pediatr. Allergy Immu. 2010; 718-722.

[5] Hellman L. Regulation of IgE homeostasis, and the identification of potential targetsfor therapeutic intervention. Biomed. Pharmacother. 2007; 61:34-49.

[6] Milian E, Diaz AM. Allergy to House Dust Mites and Asthma. Puerto Rico Health Sciences Journal 2004; 23(1):47-57. 
[7] De Blay F, Barnig C, Ott M. House dust mite control measures for asthma. Allergy 2008; 63(6):646659.

[8] Shin J-W, Sue J-H, Song T-W, Kim K-W, Kim E-S, Sohn $\mathrm{MH}$, et al. Atopy and House Dust Mite Sensitization as Risk Factors for Asthma in Children. Yonsei Med. J. 2005; 46:629-634.

[9] Noguchi E, Ohtsuki Y, Tokunaga K, YamaokaSageshima M, Ichikawa K, Aoki T, et al. ADAM33 polymorphisms are associated with asthma susceptibility in a Japanese population. Clin. Exp. Allergy 2006; 36:602-608.

[10] Lodge CJ, Lowe AJ, Gurrin LC, Hill DJ, Hosking $\mathrm{CS}$, Khalafzai $\mathrm{R}$, et al. House dust mite sensitization in toddlers predicts current wheeze at age 12 years. J. Allergy Clin. Immunol. 2011; 128:782-788.

[11] Platts-Mills T, Leung DY, Schatz M. The Role of Allergens in Asthma. Am. Fam. Physician 2007; 76:675-680.

[12] Semic-Jusufagic A, Simpson A, Woodcock A. Dust mite allergen avoidance as a preventive and therapeutic strategy. Curr. Allergy Asthma Rep. 2006; 6(6):521-526.

[13] Fernández-Caldas E, IraolaCalvo V. Mite allergens. Curr. Allergy Asthma Rep. 2005 Sep; 5(5):402-410.

[14] Ramos JDA, Castillo MPS, del Rosario M, Gapay MAS, Go TP, Kamantigue Jr EG. Allergenicity and cross-reactivity of three house dust mite species among Filipino allergic patients. Philipp. J. Sci. 2007; 136(2):139-146.

[15] Ramos JDA, Concepcion ZS, Pineda BA, Romano EL, Saep JB. Acarofauna and allergenicity of house dust mites in houses of selected Filipino allergic patients in Metro Manila. Acta Manila 2006; 54:7-14.

[16] Chmielewski W. Pollen pellets as a medium for culture of mites Suidasia pontifica (Oud.) (Acarina, Suidasiidae). J. Apic. Res 2009; 53(1):37-42.

[17] van Hage-Hamsten M, Johansson E. Clinical and immunologic aspects of storage mite allergy. Allergy 1998; 49-53.
[18] Gregory LG, Lloyd CM. Orchestrating House Dust Mite - Associated Allergy in the Lung. Trends in Immunol. 2011; 402-411.

[19] Kopp MV, Niggemann B, Forster J. House dust mite allergy: complete removal of the provoking allergen is a primary therapeutic approach. Allergy 2009; 64(1):187-188.

[20] Weghofer M, Thomas WR, Kronqvist M, Mari A, Purohit A, Pauli G, Horak F, Grönlund H, van Hage $M$, Valenta $R$, Vrtala S. Variability of IgE reactivity profiles among European mite allergic patients. Eur. J. Clin. Invest. 2008; 38(12):959-965.

[21] Fernández-Caldas E, Puerta L, Caraballo L, Lockey RF. Mite allergens. Clin. Allergy Immunol. 2008: 21:281-182.

[22] Platts-Mills TA, Rakes G, Heymann PW. The relevance of allergen exposure to the development of asthma in childhood. J. Allergy Clin. Immunol. 2000; 105:S503-8.

[23] Miraglia del Guidice M, Pedulla M, Piacentini G, Capristo C, Brunese F, Decimo F, et al. Atopy and house dust mite sensitization as risk factors for asthma in children. Allergy 2002; 169-172.

[24] Puerta L, Lagares A, Mercado D, FernandezCaldas E, Caraballo L. Allergenic composition of the mite Suidasiamedanensis and crossreactivity with Blomiatropicalis. Allergy 2005; 60:41-47.

[25] Sanchez-Borges M, Capriles-Hulett A, Fernandez-Caldas E, Suares-Chacon $\mathrm{R}$, Caballero F, et al. Mite-contaminated foods as a cause of anaphylaxis. J. Allergy Clin. Immunol. 1997; 99:738-743.

[26] Mariana A, Ho TM, Gendeh BS, Iskandar H, Zainuldin-Taib M. First report on sensitization to allergens of a house dust mite, Suidasia pontifical (Acari:Saproglyphidae). Southeast Asian Journal Tropical Med. Public Health 2000; 31:722-723.

[27] Squillace SP, Sporik RB, Rakes G, Couture N, Lawrence A, Merriam S, et al. Sensitization to Dust Mites as a Dominant Risk Factor for Asthma among Adolescent Living in Central Virginia. Am. J. Resp. Crit. Care 1997; 156:1760-1764. 